\title{
Estresse ocupacional em docentes de enfermagem: Uma revisão integrativa da
}

\section{literatura}

\author{
Occupational stress in nursing teachers: An integrative literature review
}

Estrés ocupacional en profesores de enfermería: Una revisión de la literatura integradora

Recebido: 08/04/2021 | Revisado: 16/04/2021 | Aceito: 19/04/2021 | Publicado: 04/05/2021

Cátia Aguiar Lenz

ORCID: https://orcid.org/0000-0003-1864-6988 Universidade Feevale, Brasil

E-mail: lenz@feevale.br

Michele Antunes

ORCID: https://orcid.org/0000-0002-7231-8099 Universidade Feevale, Brasil

E-mail: micheleantunes@gmail.com

Raquel de Lima dos Santos

ORCID: https://orcid.org/0000-0002-2891-0052 Universidade Feevale, Brasil

E-mail: raquel.dlds@gmail.com

Marta Rosecler Bez

ORCID: https://orcid.org/0000-0002-5542-8229

Universidade Feevale, Brasil

E-mail: martabez@gmail.com

\begin{abstract}
Resumo
A profissão do docente do ensino superior é caracterizada por sobrecargas, intensas mudanças e pressões que conduzem a diversas consequências. Objetivo foi identificar as causas do estresse ocupacional em docentes de enfermagem. Trata-se de uma revisão integrativa da literatura, nas bases de dados Literatura Latino-Americana em Ciências de Saúde (LILACS) e na Base de dados em Enfermagem (BDENF). Artigos publicados pela área da saúde, em Língua Portuguesa, no período de 2009 a maio de 2020, completos de acesso livre e online. Identificaram-se um total de 06 artigos. As discussões apresentadas pelos autores foram descritas em duas categorias. Este estudo traz como resultados os seguintes fatores e/ou situações geradoras de estresse: condições de trabalho; questões éticas; carga mental intensa; falta de reconhecimento profissional; aulas teórico-práticas; associação dos serviços de ensino e saúde; relação interpessoal; situações familiares/social, financeiras e de saúde e entre as principais complicações: estresse, ansiedade, insônia e dificuldades do sono, cefaleia, insuficiência vascular, dor, cansaço, edemas, entre outros problemas. $\mathrm{O}$ estudo evidenciou a diversidade e complexidade de diagnosticar os fatores estressores, assim como as complicações a saúde do docente de nível superior.
\end{abstract}

Palavras-chave: Enfermagem; Estresse ocupacional; Docente.

\begin{abstract}
The teaching profession of higher education is characterized by overloads, intense changes and pressures that lead to several consequences. To identify the causes of occupational stress in nursing professors. It is integrative literature review, in the Latin American Literature in Health Sciences databases (LILACS) and in the Nursing Database (BDENF). Articles published by the health area, in Portuguese, from 2009 to May 2020, complete with free and online access. A total of 06 articles were identified. The discussions presented by the authors were described in two categories. This study results in the following factors and/or situations that generate stress: working conditions; ethical issues; intense mental load; lack of professional recognition; theoretical-practical classes; association of teaching and health services; interpersonal relationship; family/social, financial and health situations and among the main complications: stress, anxiety, insomnia and sleep difficulties, headache, vascular failure, pain, tiredness, edema, among other problems. The study showed the diversity and complexity of diagnosing stressors, as well as the health complications of higher education faculty.
\end{abstract}

Keywords: Nursing; Occupational stress; Faculty.

\section{Resumen}

La profesión docente de la educación superior se caracteriza por sobrecargas, cambios intensos y presiones que conllevan varias consecuencias. El objetivo fue identificar las causas del estrés laboral en profesores de enfermería. Se trata de una revisión de literatura integradora, en las bases de datos de Literatura Latinoamericana en Ciencias de la Salud (LILACS) y en la Base de Datos de Enfermería (BDENF). Artículos publicados por el área de salud, en 
portugués, desde 2009 hasta mayo de 2020, completos con acceso gratuito y en línea. Se identificaron un total de 06 artículos. Las discusiones presentadas por los autores se describieron en dos categorías. Este estudio da como resultado los siguientes factores y / o situaciones que generan estrés: condiciones de trabajo; cuestiones éticas; carga mental intensa; falta de reconocimiento profesional; clases teórico-prácticas; asociación de servicios docentes y sanitarios; relaciones interpersonales; situaciones familiares / sociales, económicas y de salud y entre las principales complicaciones: estrés, ansiedad, insomnio y dificultades para dormir, dolor de cabeza, insuficiencia vascular, dolor, cansancio, edemas, entre otros problemas. El estudio mostró la diversidad y complejidad del diagnóstico de factores estresantes, así como las complicaciones para la salud de los docentes de educación superior.

Palabras clave: Enfermería; Estrés laboral; Maestro.

\section{Introdução}

O trabalho é considerado fundamental nas atividades diárias das pessoas, pois através dele é possível transformar a realidade, viabilizar a sobrevivência e formar a identidade. Personificação do ser humano ocorre por meio do exercício profissional, no qual sente-se digno e integrante da sociedade. Grande parte das pessoas dedicam seu tempo ao trabalho e esta experiência se estende para além do ambiente laboral, na medida em que se reflete nos processos de inserção social e na saúde dos trabalhadores (Codo \& Vasques-Menezes, 2002; Dejours, 1992; Marx, 1985; Sampaio, 2002).

$\mathrm{Na}$ atualidade o estresse é considerado um problema e de fato interfere na qualidade de vida das pessoas, ocasionando danos físico, psicológico, profissional e social (Camargo et al., 2013). É definido como um conjunto de alterações psicofisiológicas, que ocorrem como resposta do organismo ao sofrer com determinadas situações que o preparam para a batalha ou para a fuga que pode afetar a saúde física e psicológica, a qualidade de vida pessoal e profissional e a sensação de bem-estar como um todo (Lipp, 2001).

A profissão do docente do ensino superior é caracterizada por sobrecargas, intensas mudanças e pressões que conduzem a diversas consequências, tais como, estresse, excesso de preocupação com a sua profissão e com a necessidade de atualizações educacionais constantes. Estes componentes podem induzir queda na qualidade de vida emocional, com aumento da pressão em nível físico e psicológico que conduzem a sintomas de estresse intenso (Silva \& Coltre, 2009). Dentre as fontes de estresse no trabalho, estão o excesso de atividades, os conflitos de interesse, dificuldades interpessoais e de lidar com as cobranças, bem como a falta de cooperação e de reconhecimentos e os problemas administrativos (Sadir \& Lipp, 2009).

Quando o docente do ensino superior é da área da saúde, o problema também é evidenciado pelo profissional não possuir em sua formação na graduação disciplinas voltadas para a docência e formação pedagógica, além de muitos virem de uma atividade assistencial, ou seja, da prática clínica, gerando estresse na transição desta para o ensino (Moran, 2012). Diante do exposto, esta pesquisa objetivou identificar as causas do estresse ocupacional em docentes de enfermagem.

\section{Metodologia}

Este estudo trata-se de uma revisão integrativa da literatura (Cooper, 1982) estruturada em cinco etapas, tais como: formulação do problema, coleta dos dados, avaliação dos dados coletados com análise qualitativa e interpretação e apresentação dos resultados.

Neste estudo aplicou-se a metodologia da pesquisa-ação, de forma qualitativa, no qual procurou evidenciar nas literaturas relacionadas meios para a solução do problema exposto, a partir do exercício reflexivo a qual se investigou através da leitura, selecionou e, posteriormente, avaliou-se as informações para obtenção e análise dos dados, conforme a compreensão e utilização das normas exigidas de acordo com o tipo de estudo (Pereira et al., 2018).

Deste modo, a questão norteadora desta revisão integrativa foi: quais são as causas do estresse ocupacional em docentes de enfermagem?

A coleta dos dados foi realizada nas bases de dados Literatura Latino-Americana em Ciências de Saúde (LILACS) e na Base de dados em Enfermagem (BDENF). Os critérios de inclusão foram: artigos completos de acesso livre, online e 
gratuitos que contenham como Descritores em Ciências da Saúde (DeCS): "enfermagem", "estresse ocupacional", "docentes", fazendo uso de uma string de busca "AND”, desenvolvidos pela área da saúde, publicados em Língua Portuguesa, no período de 2009 a 2020. E como critérios de exclusão foram: artigos duplicados, estudos de revisão, literatura cinzenta, livros, resumos, editoriais, monografias, dissertações e teses.

Para avaliação dos dados foi confeccionado um instrumento de coleta de dados, onde consta os dados de identificação e informações de cada artigo. À medida que foi realizada a leitura aprofundada dos conteúdos, foi realizada a avaliação dos dados de forma coerente e organizada, buscando esclarecimento sobre o tema e a problematização do estudo para posterior análise e interpretação dos resultados obtidos. Para apresentação dos dados e seus critérios de escolha fez-se o uso de uma ferramenta denominada Prisma (Moher et al., 2009), conforme fluxograma 1.

Além disso, seguindo os critérios metodológicos de Cooper (1982), os artigos selecionados foram organizados em um quadro sinóptico, o que permitiu a sintetização das informações e, posteriormente, foram sumarizados para a definição de categorias/grupos de discussão temática de modo a apresentar os resultados similares e buscar explicações para os resultados discordantes.

\section{Resultados e Discussão}

Identificou-se o total de 20 artigos, que após a aplicação dos critérios de inclusão e de exclusão, foram avaliados, conforme a exemplificação do processo no Fluxograma, Figura 1.

Figura 1 - Fluxograma do processo de seleção dos estudos para revisão.

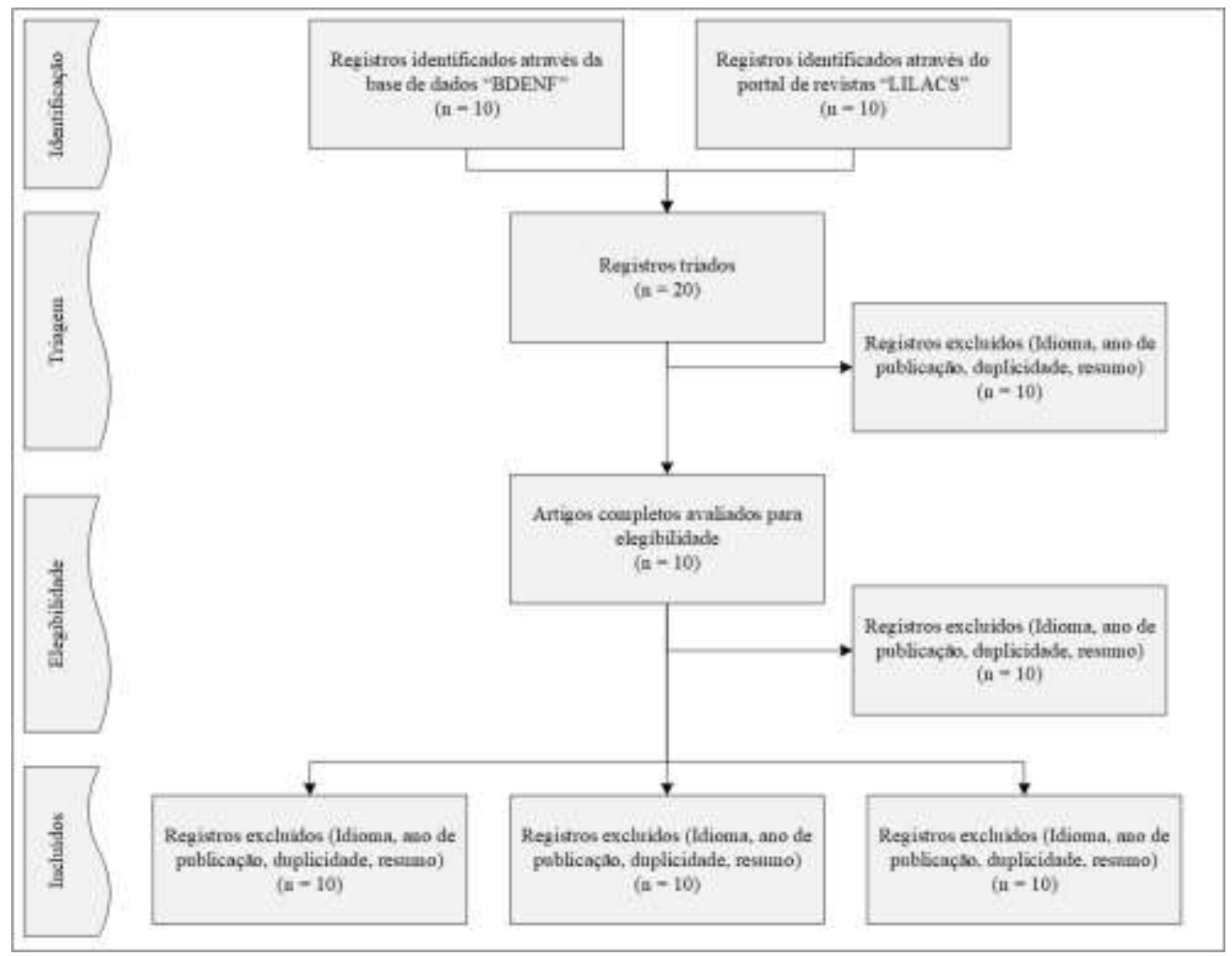

Fonte: Autoras (2020). Prisma Statement adaptado de Moher et al. (2009).

O fluxograma acima tem o objetivo de relatar a seleção dos artigos que compuseram a amostra deste estudo, demonstrando as etapas de forma objetiva, com as fases de identificação dos artigos nas bases de dados; seleção destes, através de uma triagem e com a exclusão daqueles que estavam duplicados, em forma de resumo, idioma ou ano de publicação em 
desacordo com os critérios de seleção; logo, foram avaliados a elegibilidade e novamente excluídos os estudos em que o tema não estavam condizentes com a pesquisa e por fim incluindo-se os estudos de síntese qualitativa e quantitativa conforme os critérios de inclusão previamente indicados.

A amostra final constitui-se de 06 artigos, destes, 03 foram publicados na base de dados da BDENF e 03 na LILACS, sendo todos escritos em português. O Quadro 1 sintetiza os artigos que compuseram o estudo, destacando as informações sobre o título, autor, ano e base de dados selecionada, sendo que, para melhor organização, cada um dos artigos foi identificado com um número.

Quadro 1 - Quadro sinóptico: distribuição dos artigos que compuseram este estudo.

\begin{tabular}{|c|c|c|c|c|c|}
\hline $\mathbf{N}^{\mathbf{o}}$ & Título do artigo & Autores & Ano & $\begin{array}{c}\text { Base de } \\
\text { dados }\end{array}$ & $\begin{array}{l}\text { Tipo de } \\
\text { Estudo }\end{array}$ \\
\hline 1 & $\begin{array}{l}\text { Processo de trabalho e disposição ao } \\
\text { estresse entre docentes de ciências } \\
\text { biológicas e da saúde }\end{array}$ & $\begin{array}{l}\text { Fadel, C., Flores, M.T., Brigola, } \\
\text { S., Zanesco, C., Bordin, D., \& } \\
\text { Martins, A.S. }\end{array}$ & 2019 & LILACS & $\begin{array}{l}\text { Quali- } \\
\text { quantitativo }\end{array}$ \\
\hline 2 & $\begin{array}{l}\text { Trabalho docente de enfermagem e } \\
\text { as repercussões no processo saúde- } \\
\text { doença }\end{array}$ & $\begin{array}{l}\text { D'Oliveira, C.A.F.B., Almeida, } \\
\text { C.M., Souza, N.V.D.O., Pires, } \\
\text { A., Madriaga, L.C.V., \& } \\
\text { Varella, T.C.M.Y.M.L. }\end{array}$ & 2018 & LILACS & Qualitativo \\
\hline 3 & $\begin{array}{l}\text { Docência universitária e o estresse: } \\
\text { estressores nos cursos de } \\
\text { enfermagem e medicina }\end{array}$ & $\begin{array}{l}\text { Santos, N.P., Marinho, F.P., } \\
\text { Lima, K.Y.N., Rodrigues, } \\
\text { C.C.F.M., \& Santos, V.E.P. }\end{array}$ & 2016 & B-DENF & Qualitativo \\
\hline 4 & $\begin{array}{l}\text { Riscos ocupacionais psicossociais e } \\
\text { sua repercussão na saúde de } \\
\text { docentes universitários }\end{array}$ & $\begin{array}{l}\text { Caran, V.C.S., Freitas, F.C.T., } \\
\text { Alves, L.A., Pedrão, L.J., \& } \\
\text { Robazzi, M.L.C.C. }\end{array}$ & 2011 & B-DENF & Quantitativo \\
\hline 5 & $\begin{array}{l}\text { Docência em Enfermagem: } \\
\text { insatisfações e indicadores } \\
\text { desfavoráveis }\end{array}$ & $\begin{array}{l}\text { Corral-Mulato, S., Bueno, } \\
\text { S.M.V., \& Franco, D.M. }\end{array}$ & 2010 & LILACS & Qualitativo \\
\hline 6 & $\begin{array}{l}\text { O estresse nos docentes de } \\
\text { enfermagem de uma universidade } \\
\text { pública }\end{array}$ & $\begin{array}{l}\text { Miranda, L.C.S., Pereira, C.A., } \\
\& \text { Passos, J.P. }\end{array}$ & 2009 & B-DENF & $\begin{array}{l}\text { Quali- } \\
\text { quantitaivo }\end{array}$ \\
\hline
\end{tabular}

Fonte: Autoras (2020).

O quadro acima tem o intuito de apresentar os artigos que compuseram a amostra, destacando o título, autoria, ano de publicação, base de dados e tipo de estudo.

Após a leitura e análise dos artigos, foram identificadas as principais discussões mencionadas que influenciam as a causas do estresse ocupacional em docentes de enfermagem e as consequências à saúde do professor. Estas foram agrupadas em duas categorias, sendo elas e seus respectivos artigos:

a) categoria 1: Fatores e/ou situações geradoras de estresse nos docentes de nível superior em enfermagem; que aborda os principais fatores estressores laborais no professor de enfermagem das Instituições de Ensino Superior (IES); artigos de 1 a 6 ;

b) categoria 2: Consequências à saúde dos docentes de nível superior em enfermagem relacionados aos fatores e/ou situações geradoras de estresse; que aborda as principais enfermidades laborais ocorridas como consequência do estresse no professor de enfermagem das instituições de Ensino Superior (IES); artigos de 1 a 6. 
A análise dos artigos selecionados nos permitiu identificar os que se referem as categorias 1 e 2, com 06 artigos cada. Destaca-se que um mesmo estudo poderia atender mais de uma categoria.

\section{Categoria temática 1: fatores e/ou situações geradoras de estresse nos docentes de nível superior em enfermagem}

No artigo 1 de Fadel et al. (2019) os fatores determinantes de estresse nos docentes foram as reuniões, os prazos, burocracia, cobranças, problemas administrativos; atrito com estudantes e docentes; falta de infraestrutura e funcionários; injustiças; atividades rotineiras, estilo de vida.

O artigo 06 de Miranda et al. (2009), traz resultados semelhantes, pois destacam as condições de trabalho e referem-se principalmente aos insuficientes recursos humanos e materiais para a realização das atividades pedagógicas, bem como o volume de trabalho/ excesso de atividades; relações profissionais/desagregação em ambiente de trabalho; problemas políticoadministrativos no trabalho; estudos (qualificação); reuniões e problemas com os alunos; e trabalho noturno e em fins de semana.

Os docentes ainda citaram o excesso de trabalho, de cobrança e responsabilidade, a urgência de assuntos, a dificuldade no cumprimento do planejamento de tarefas, aliado à crescente burocratização e ao estresse cotidiano, como geradores de momentos de insatisfação profissional, e, consequentemente, a falta de tempo, para que o profissional possa descansar, divertir-se e, até mesmo, estar com seus familiares conforme os estudos de Corral-Mulato et al. (2010), artigo 05.

D'Oliveira et al. (2018), artigo 02, discorrem que, com o quadro profissional reduzido, os docentes vêm-se obrigados a acumular cargos, funções e atividades, corroborando com a polivalência e a multifuncionalidade. A competição, as assessorias, consultorias, atividades de graduação e pós-graduação, os prazos curtos e excesso de atividades facilitam a ocorrência do estresse e manifestações a ele agregadas, Caran et al. (2011), artigo 04.

Ainda Santos et al. (2016), artigo 03, destacam que apesar da relação importante entre o desgaste físico e o estresse laboral, outro fator apresentado como relevante e, muitas vezes, como o principal, foi o estresse psicológico. No estudo supracitado, os docentes destacaram: a falta de habilidade dos alunos; a responsabilidade com a assistência adequada ao paciente ao mesmo tempo em que orienta e ensina o aluno nos campos de prática; a alta demanda de atividades e procedimentos; a grande quantidade de alunos por grupo em alguns campos de estágio; e lidar com a dor e sofrimento dos pacientes.

Para D'Oliveira et al. (2018), artigo 02, os serviços assistenciais nos quais ocorrem os campos de estágio dos discentes da universidade, os fatores dificultadores para a execução do trabalho, estão relacionados à falta de recursos materiais, estrutura física e a falta de acompanhamento de recursos tecnológicos.

Para os autores, Miranda et al. (2009), no artigo 06 as situações sociais e familiares geradoras de estresse mencionadas pelos docentes em sua pesquisa tais como: falta de tempo e intimidação/cobrança/pressão; cansaço; transporte, violência, casa e família; ritmo acelerado e acúmulo de tarefas. Destaca-se ainda que para o docente dar conta desta demanda laboral, por vezes, necessita utilizar seu tempo de descanso e lazer, renunciando da convivência com a família e amigos, ou opta por abdicar de fazer alguma atividade em prol de sua saúde (D'Oliveira et al., 2018), artigo 02.

Portanto, constatou-se que as dificuldades dos docentes para desenvolverem o processo laboral estão fortemente atreladas a configuração do mundo do trabalho, que tem sido pautado pela lógica da produtividade, que captura a subjetividade do trabalhador de forma engenhosa e o faz trabalhar sob ritmo intenso para dar conta da multiplicidade de tarefas (D'Oliveira et al., 2018), artigo 02. 


\section{Categoria temática 2: consequências à saúde dos docentes de nível superior em enfermagem relacionados aos fatores e/ou situações geradoras de estresse}

As demandas da organização do trabalho requeridas aos trabalhadores, como: polivalência, multifuncionalidade, intensificação dos ritmos de trabalho e sobrecarga laboral; faz com que os docentes refiram cansaço, esgotamento físico e mental, além de se sentirem estressados conforme D'Oliveira et al. (2018), artigo 02.

$\mathrm{Na}$ pesquisa de Fadel et al. (2019), artigo 1, as alterações verificadas nos docentes foram modificações físicas e psicológicas, nas quais houve alteração hormonal, insônia; cansaço corporal; exaustão intelectual; Fatores procedentes: psicológicos como alteração emocional e psicológica inespecífica, ansiedade e irritabilidade.

Nos estudos de Miranda et al, (2009), artigo 06, onde aplicaram um teste para verificar a probabilidade de os professores adoecerem, ou seja, as pessoas com maior risco de adoecer e concluíram que $70 \%$ dos sujeitos têm um alto nível de estresse e técnicas ineficientes para lidar com esta situação. Os autores destacam ainda que níveis elevados de estresse podem predispor a um risco de $50 \%$ de adoecimento.

Nessa revisão, observa-se que as consequências mencionadas pelos autores estão associadas como uma "cascata" de complicações. Nota-se nos estudos de Caran et al. (2011), artigo 04, que a manifestação predominantemente apontada foi o estresse seguido de ansiedade, insônia e dificuldades do sono e cefaleia. O estresse foi a alteração na saúde mais mencionada pelos docentes e agregada às seguintes manifestações: depressão, ansiedade, mau humor, insônia, fadiga, irritabilidade, alteração da glicose, cefaleia, dispneia, desânimo, desconfiança, cansaço físico e mental, alterações da pressão e taquicardia.

A ansiedade foi apontada pelos sujeitos, os quais a associaram com desmotivação, gastrite, irritabilidade, estresse, insônia, pouca tolerância, nervosismo, cefaleia, tristeza, desânimo, angústia, falta de criatividade, insatisfação, cansaço, falta de concentração, insegurança, indisposição, alterações da memória e hábitos alimentares, pressão, isolamento, diarreia, resfriados frequentes, taquicardia e dor de estômago. Este é um quadro sintomatológico do estresse e ocorre dependendo da fase em que ele se encontra (Santos et al., 2016), artigo 03.

Os autores Fadel et al. (2019), artigo 1, verificaram que o estresse impacta psicologicamente o docente, interferindo em suas relações e na qualidade do trabalho. Dentre os fatores psicológicos, verificou-se a falta de concentração, desânimo e impaciência. Nas relações, os aspectos como baixa qualidade na relação com estudantes, convívio ruim com funcionários e colegas de trabalho, estiveram presentes. E quanto ao impacto no processo de trabalho, itens como a queda na qualidade e desempenho, diminuição da disposição, motivação e dificuldade para a tomada de decisão, foram identificados.

Ainda para Santos et al. (2016), artigo 03, os docentes apresentaram, doenças e complicações como, insuficiência vascular, dor, cansaço e edemas, possivelmente associados à rotina de trabalho. Seja com relação às aulas, a prática com alunos, ou até mesmo os plantões, os docentes afirmaram reconhecer os diversos efeitos físicos decorrentes do estresse de seus corpos, muitas vezes, os impedindo de realizar suas atividades de forma satisfatória. Soma-se a essa carga de funções docentes, os ambientes inadequados onde ocorrem as aulas práticas e os estágios supervisionados, que são atividades descritas como as mais estressantes pelos docentes.

Nos estudos de D'Oliveira et al. (2018), artigo 02, na fala dos participantes, as maiores repercussões no processo saúde-doença apontados foram o cansaço, o esgotamento físico e mental e o estresse. Neste sentido, verifica-se que a forma como a organização do trabalho se configura, repercute negativamente na saúde, no convívio familiar e social.

Os dilemas, se não forem bem trabalhados, discutidos, revistos e modificados na busca de um melhor ambiente laboral, poderão levar o profissional a desenvolver problemas de saúde, tanto físicos, como mentais (Corral-Mulato et al., 2010), artigo 05. Cabe destacar ainda que, em meio a tantos estressores, faz-se necessário cuidar da saúde física e mental destes docentes, a fim de evitar impactos, tanto individuais, quanto organizacionais (Santos et al., 2016), artigo 03. 
Por fim, notou-se que nos estudos não foram destacados pelos professores estratégias de enfrentamento para redução do estresse, mesmo não sendo objetivo deste considera-se importante tal reconhecimento. Sabe-se que melhorias na gestão do trabalho, no processo de ensino e nas estratégias de enfrentamento pessoal são alternativas para redução do estresse e podem produzir resultados mais rápidos e eficazes na promoção da saúde (Stokols, 1992; Dalagasperina \& Monteiro, 2016).

\section{Considerações Finais}

Acredita-se que o presente estudo atingiu seu objetivo uma vez que foi possível responder à questão de pesquisa: “quais são as causas do estresse ocupacional em docentes de enfermagem?", através da identificação nas produções científicas.

$\mathrm{Na}$ categoria 1, pode-se identificar os fatores e/ou situações geradoras de estresse nos docentes de nível superior em enfermagem e na categoria 2 , sobre as consequências à saúde dos docentes de nível superior em enfermagem relacionados aos fatores e/ou situações geradoras de estresse.

O presente estudo mostrou-se relevante devido a identificação dos fatores geradores de estresse e as consequências a saúde dos professores do curso de graduação de enfermagem. As discussões realizadas no estudo mostraram a diversidade e a complexidade de diagnosticar os fatores estressores e as complicações a saúde do docente de nível superior do curso de enfermagem.

Portanto diante de tais resultados, nota-se que a busca fez com que as autoras obtivessem uma seleção pequena de estudos que abordaram o assunto, o que evidencia uma carência de artigos publicados gratuitamente sobre o tema. Almeja-se que a comunidade acadêmica e científica dediquem-se à produção de estudos futuros, de preferência, relacionados à pesquisa de campo com os profissionais enfermeiros e docentes a fim de buscar conhecer e compreender, em pesquisas atuais, o estresse, os fatores estressores, bem como as estratégias que utilizam para o enfrentamento destes no ensino superior pela importância e magnanimidade que apresentam para a saúde e qualidade de vida destes profissionais.

\section{Referências}

Camargo, E., Ponestki Oliveira, M., Rodriguez-Añez, C., Akira Ferreira Hino, A., \& Siqueira Reis, R. (2013). Estresse percebido, comportamentos relacionados à saúde e condições de trabalho de professores universitários. Psicologia Argumento, 31(75). 589-597.

Codo, W., \& Vasques-Menezes, I. (2002). Educar, educador. In W. Codo (Org.), Educação: carinho e trabalho, Vozes.

Caran, V. C. S., Freitas, F. C. T., Alves, L. A., Pedrão, L. J., \& Robazzi, M. L. C. C. (2011). Riscos ocupacionais psicossociais e sua repercussão na saúde de docentes universitários. Rev Enferm UERJ, 19(2), 255-261. https://pdfs.semanticscholar.org/da93/787f3ba2f607f350cd16eeea169d18cf5b77.pdf

Cooper, H. M. (1982) Scientific guidelines for conducting integrative research reviews. Rev Educ Res, 52(2), 291-302. https://doi.org/10.3102\%2F00346543052002291

Corral-Mulato, S., Bueno, S. M. V., \& Franco, D. M. (2010) Docência em Enfermagem: insatisfações e indicadores desfavoráveis. Acta Paul Enferm., 23(6), 769-774. http://dx.doi.org/10.1590/S0103-21002010000600009

Dalagasperina, P., \& Monteiro, J. K. (2016). Estresse e docência: um estudo no ensino superior privado. Revista Subjetividades, 16(1), 3651. https://dx.doi.org/10.5020/23590777.16.1.37-51

Dejours, C. (1992). A Loucura no Trabalho: estudo da psicopatologia do trabalho. Cortez Oboré

D'Oliveira, C. A. F. B., Almeida, C. M., Souza, N. V. D. O., Pires, A., Madriaga, L. C. V., \& Varella, T. C. M. Y. M. L. (2018) Trabalho docente de enfermagem e as repercussões no processo saúde-doença. Rev Fund Care Online, 10(1), 196-202. http://dx.doi.org/\%2010.9789/2175-5361.2018.v10i1.196202

Fadel, C. B., Flores, M. T., Brigola, S., Zanesco, C., Bordin, D., \& Martins, A. S. (2019) Processo de trabalho e disposição ao estresse entre docentes de ciências biológicas e da saúde. Rev Fun Care Online, 11(4), 843-848. http://www.seer.unirio.br/index.php/cuidadofundamental/article/view/6666/pdf_1

Lipp, M. E. N. (2001). Estresse emocional: a contribuição de estressores internos e externos. Revista de Psiquiatria Clínica, 28(6), $347-349$.

Marx, K. (1985). O capital. Nova Cultura.

Miranda, L. C. S., Pereira, C. A., \& Passos, J. P. (2009) O estresse nos docentes de enfermagem de uma universidade pública. Rev Fund Care Online, 1(2), 335-344. http://www.seer.unirio.br/index.php/cuidadofundamental/article/view/410 
Research, Society and Development, v. 10, n. 5, e20710514791, 2021

(CC BY 4.0) | ISSN 2525-3409 | DOI: http://dx.doi.org/10.33448/rsd-v10i5.14791

Moher, D., Liberati, A., Tetzlaff, J., Altman, D. G., \& The PRISMA Group. (2009) Preferred reporting items for systematic reviews and meta analyses: The Prisma statement. PLoS Med., 6(7), e1000097. https://journals.plos.org/plosmedicine/article?id=10.1371/journal.pmed.1000097

Moran, R. (2012) Retention of new graduate nurses. J Nurses Staff Dev, 28(6), 270-273. https://doi.org/10.1097/NND.0b013e318272584a

Pereira, A. S., Parreira, F. J., Shitsuka, D. M., \& Shitsuka, R. (2018). Metodologia da pesquisa científica. UFSM. https://repositorio.ufsm.br /bitstream/handle/1/15 824/Lic_Computacao_Metodologia-Pesquisa-Cientifica.pdf?sequence=1.

Sadir, M. A., \& Lipp, M. E. N (2009). As fontes de stress no trabalho. Revista de Psicologia da IMED, 1(1), 114-126. https://www.researchgate.n et/publication/24 2191562_As_Fontes_de_Stress_no_Trabalho

Santos, N. P., Marinho, F. P., Lima, K. Y. N., Rodrigues, C.C. F. M. \& Santos, V. E. P. (2016) Docência universitária e o estresse: estressores nos cursos de enfermagem e medicina. Rev Enferm UFSM, 6(1), 61-70. https://doi.org/10.5902/2179769217078

Sampaio, J. J., \& Messias, E. L. (2002). A epidemiologia em saúde mental e trabalho. In M. G. Jaques, \& W. Codo (Orgs.), Saúde mental \& trabalho (pp.143172). Vozes.

Silva, S. F. P. \& Coltre, S. M. (UFSC). (2009). O nível de estresse dos docentes da área da saúde em uma instituição de ensino superior privada no oeste do Paraná. IX Colóquio Internacional Sobre Gestão Universitária na América do Sul. http://repositorio.ufsc.br/xmlui/handle/123456789/36862

Stokols, D. (1992). Establishing and maintaining health environments. Toward a social ecology of health promotion. American Psychologist, 47, 6-22. https://pubmed.ncbi.nlm.nih.gov/1539925/ 